\title{
Mechanism of active site exclusion in a site-specific recombinase role of the DNA substrate in confering half-of-the-sites activity
}

\author{
Jehe Le, Takashi Tonozuka, ${ }^{1}$ and Makkuni Jayaram ${ }^{2}$ \\ Department of Microbiology and Institute of Cell and Molecular Biology, University of Texas at Austin, \\ Austin, Texas 78712 USA
}

\begin{abstract}
The Flp site-specific recombinase assembles its active site by recruiting the catalytic tyrosine (Tyr-343) from one Flp monomer into the pro-active site containing a triad of Arg-191, His-305, and Arg-308 from a second monomer. In principle, two active sites may be assembled from a Flp dimer by simultaneous, reciprocal contribution of the shared amino acids by its constituent monomers. In practice, only one of the two active sites is assembled at a time, as would be consistent with a recombination mechanism involving two steps of single-strand exchanges. By using substrates containing strand-specific base bulges, we demonstrate that the relative disposition of their DNA ams can account for this active site exclusion. We also show that the exclusion mechanism operates only at the level of positioning Tyr-343 with respect to the pro-active site, and not at the level of orienting the labile phosphodiester bond within the DNA chain. It is not negative cooperativity of substrate binding but, rather, the substrate-induced negative cooperativity in protein orientation that accomplishes half-of-the-sites activity in the Flp system.
\end{abstract}

[Key Words: DN A recombination; active site assembly; nucleotide bulges; DN A conformation; catalytic complementation]

Received July 17, 1997; revised version accepted September 5, 1997.

Achieving spatial and temporal coordination of chemical bond breakage and formation is a challenge faced by multisubunit enzyme systems that accomplish one round of the final reaction through more than one chemical step. Phosphoryl transfer reactions in nucleic acids, RNA splicing, DNA transposition, and DNA recombination present this case. The crux of the problem is exemplified by conservative, site-specific recombination between two DNA partners, in which four chemically identical phosphodiester bonds are broken and joined by four subunits of the recombinase enzyme. The only biologically meaningful outcome is determined by a unique configuration of the breakage-joining events.

Two pathways of conservative site-specific recombination have been shown (Stark et al. 1992; Jayaram 1994; Sadowski 1995). Recombinases that belong to the invertase/ resolvase family make doubl e-strand breaks in partner substrates and exchange strands in a concerted onestep reaction. Recombinases of the integrase (Int) family make single-strand cuts and complete recombination in

\footnotetext{
${ }^{1}$ Present address: University of Shizuoka, 52-1 Yada, Shizuoka 422, Ja pan.

${ }^{2}$ Corresponding author.

E-MAIL jayaram@almach.cc.utexas.edu; FAX (512) 471-5546.
}

two steps of single-strand exchanges. A Holliday junction is therefore an obligatory intermediate during Int family recombination. In both types of recombination, strand cleavage and strand joining are transesterification reactions, the former being executed by a recombinasederived nucleophile (an active site serine or tyrosine) and the latter by a DN A-derived nucleophile (a 5' or 3' hydroxyl group exposed by strand breakage). The conservation of the phosphodiester bond during strand cutting, by linkage of the broken DNA end to the recombinase, eliminates the requirement for an exogenous energy source for the progression of the reaction. The reaction proceeds without addition or removal of nucleotides, and yields products that are, except for their recombined configuration, chemically and energetically equivalent to the substrates.

The FIp site-specific recombinase from Saccharomyces cerevisiae follows the Int family mechanism (Fig. 1A). A monomer of Flp (the native state of the protein in solution) can bind to its recognition sequence within the recombination target site, but is a catalytically inert entity. The assembly of a functional active site requires amino acid contributions from two Flp monomers occupying the oppositely oriented binding elements that flank the strand exchange region (or spacer) (Chen et al. 


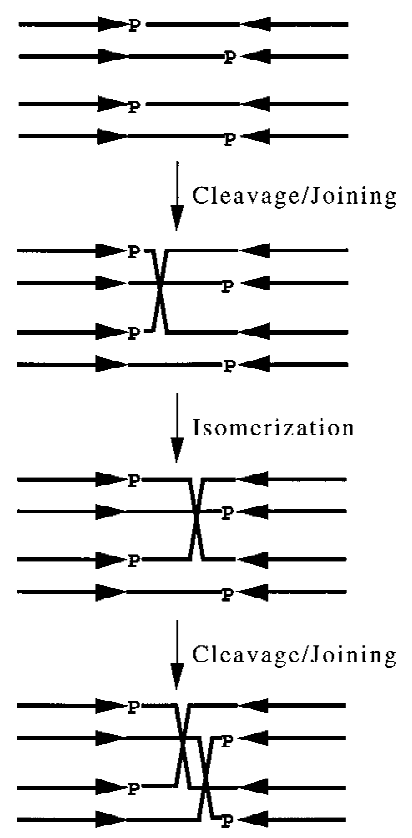

$\Lambda$
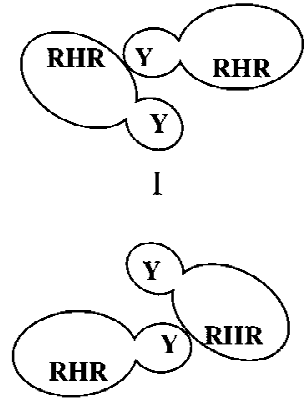

II

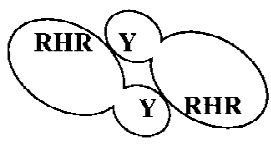

III

Tyr-343 of Flp correspond to the four invariant signature residues of the Int family (Argos et al. 1986; Abremski and Hoess 1992).

Consistent with the two-step exchange mechanism, a dimer of FIp assembled on a DN A substrate is functionally asymmetric and yiel ds DNA cl eavage al most exclusively at either one (but not both) of the scissile phosphodiester bonds that define the limits of the spacer on the two DN A strands (Qian et al. 1990; cumulative results from our laboratory and the Cox and Sadowski laboratories). Therefore, of the two possible cleavage pockets that can be derived from a pair of Flp monomers, one is excluded at any particular time. In this paper we demonstrate that the constraints imposed by the structure of the spacer on the relative stacking of the Flpbound DNA arms can provide the mechanism for this active site exclusion. When the spacer constraint is re laxed, active site exclusi on can be overcome. Therefore, the DNA substrate is the sole agent for "half-of-the sites" activity of Flp.

\section{Results}

Figure 1. Strand-exchange mechanism and mode of active site
assembly by the Flp recombinase. (A) The two-step, pairwise strand-exchange mechanism for the Flp recombination reaction (and, in general, for the Int family reaction) is shown schematically. The Flp-binding elements on each substrate are depicted by the parallel arrows arranged in a head-to-head configuration. Strand breakage and exchange at one end of the spacer (e.g., the left end) by a pair of active sites generates the Holliday junction. During this step, active site assembly at the right end is proscribed. Isomerization of the junction permits the assembly of active sites at the right to resolve it into recombinant products. During this step, active site organization at the left end is forbidden. (B) In the context of the shared active site of Flp, the first step of the reaction corresponds to the asymmetric Flp dimer configuration shown in I. Here, the RHR triad is provided by the Flp monomer at the left, and the active site tyrosine (Tyr-343) by the monomer at the right. In this assembly state, the reciprocal mode of sharing (shown in II; RHR triad from the right monomer and Tyr-343 from the left monomer) is prohibited. The assembly of II required for the second step (resolution) disrupts the initial sharing mode. The symmetric dimer shown in III, which simultaneously accommodates I and II, is contradicted by experimental evidence. Double-stranded cleavage is not observed in a DNA substrate occupied by a Flp dimer.

1992b; Lee and Jayaram 1993; Pan et al. 1993; Lee et al. 1994). One of the monomers donates the pro-active site containing the catalytic triad of Arg-191, His-305, and Arg-308 (the RHR triad), whereas the second monomer donates the active site nucleophile (Tyr-343), toward one-strand cleavage reaction. The RHR triad donor, even one that lacks Tyr-343, can facilitate the strand-joining reaction by using the $5^{\prime}$-hydroxyl group from the Flpcleaved partner DNA to attack the 3'-phosphotyrosine bond formed at the cleavage site. The RHR triad and

The three possible modes of association between two FIp monomers that generate one or two strand cleavage pockets are shown schematically in Figure 1B. Dimerization of Flp occurs only after the protein has bound to DNA. Available experimental evidence is consistent with an asymmetric dimer in which the two active sites are exclusive (I and II, Fig. 1B), and disfavors the symmetric dimer in which they are inclusive (III, Fig. 1B). The relative orientation of the Flp-binding el ements and the 8-bp spacing between them in a minimal "full-site" substrate (containing two binding arms; see, e.g., Serre et al. 1992) places two DNA-bound Flp monomers on nearly opposite faces of the B-form double helix and roughly $30 \AA$ apart (Panigrahi and Sadowski 1994; Kimball et al. 1995). The spacer DNA is free of extensive protein occupancy, as inferred from a number of footprinting experiments. Therefore, to establish an interface between the monomers for assembling the shared active site, the spacer DNA must be distorted in some way. The Flp dimer introduces a large "bend" within the spacer ( $>140^{\circ}$, called the type II bend), as measured by gel mobility against bent-DN A standards (Schwartz and Sadowski 1990; Chen et al. 1992a; Luetke and Sadowski 1995). If DNA bending is the substrate's way of accommodating catalytically rel evant Flp-FIp interactions (as was first postulated by Chen et al. 1992a), the geometry of the bend could, in principle, restrict active site assembly by the Flp dimer. For example, the center of the bend and its rel ative direction might impose a choice of one of two functionally equival ent dimer interfaces. The assembly of one active site would then be possible only at the expense of the second, therefore providing the basis for a reaction mechanism that uses single-strand exchanges. We have tested this idea with the help of syn- 
thetic substrates in which the stacking of the Flp-binding arms is constrained in a directed fashion.

Strand cleavage in synthetic full sites containing strand-specific base bulges

Bulges in DNA created by the presence of one or more nucleotides in one strand, unopposed by nucleotides in the second strand, introduce kinking or bending of the helix at the site of the bulge (Rosen et al . 1992; Gohlke et al. 1994). The bending forces the DN A arms flanking the bulge to tilt away from it. In addition, the relative twist between the two base pairs separated by the bulge is likely to be significantly greater than that for two neighboring base pairs in normal, unbulged DNA. Fluorescence resonance energy transfer (FRET) and solution nuclear magnetic resonance (N M R) studies indicate that the magnitude of the bulge-induced bend is $\sim 50-70^{\circ}$ for a three-base bulge $\left(A_{3}\right.$ or ATA ) and $85-105^{\circ}$ for a five-base bulge $\left(A_{5}\right)$. The bulged substrates that we used in our assays contain either $A_{3}$ or $A_{5}$ bulges.

Strand cleavages mediated by wild-type FIp and two Flp variants, FIp(H305L) and FIp(Y60S), in substrates containing $\mathrm{A}_{3}$ and $A_{5}$ bulges, respectively, within the spacer on the top or the bottom strand are shown in Figure 2. Flp(H305L) is a Flp variant that can mediate cleavage in a full site, but is severely impaired in the joining of cleaved ends (Parsons et al. 1988; Lee and Jayaram 1993; Pan et al. 1993). Flp(Y60S) is a mutant that is incompetent in DNA bending and extremely inefficient in cleaving a normal full site (Chen et al. 1991). When the substrate was unbulged (containing the normal 8-bp spacer; Fig. 2, lanes 1-4), cleavage by Flp occurred at either the top strand ( $C L$; left side cl eavage) or the bottom strand ( $C R$; right side cleavage) (Fig. 2, lane 2). There was no strong bi as toward one or the other type of cleavage. Consistent with earlier results, the levels of cleavage were elevated with Flp(H305L) (Fig. 2, lane 3), and only a trace amount of strand cutting was observed with FIp(Y60S) (Fig. 2, Iane 4). As noted repeatedly in previous experiments, fractionation of the reactions in native gels did not indicate the presence of the doubly cleaved product with any of the three proteins (data not shown). When the substrate contained an $A_{3}$ bulge on the top strand (Fig. 2, lanes 5-8), two striking results were observed. First, FIp(Y 60S) showed easily detectable levels of the cleavage product (Fig. 2, lane 8). Second, the cleavage was no longer unbiased, as FIp, FIp(H305L), and $\mathrm{FIp}(\mathrm{Y} 60 \mathrm{~S})$ yielded predominantly top-strand cleavage (CL) (Fig. 2, lanes 6-8). When the $A_{3}$ bul ge was located on the bottom strand of the substrate (Fig. 2, lanes 9-12), the cleavage asymmetry was reversed, with the reaction being directed almost exclusively to the bottom strand (CR) (Fig. 2, lanes 10-12). The strand cleavage bias and the bias directionality were reproducible when the $A_{3}$ bulges were replaced by $A_{5}$ bulges (Fig. 2, lanes 13-20). When the bulges were balanced on both strands (Fig. 2, lanes 21-24), approximate cleavage parity, as seen with
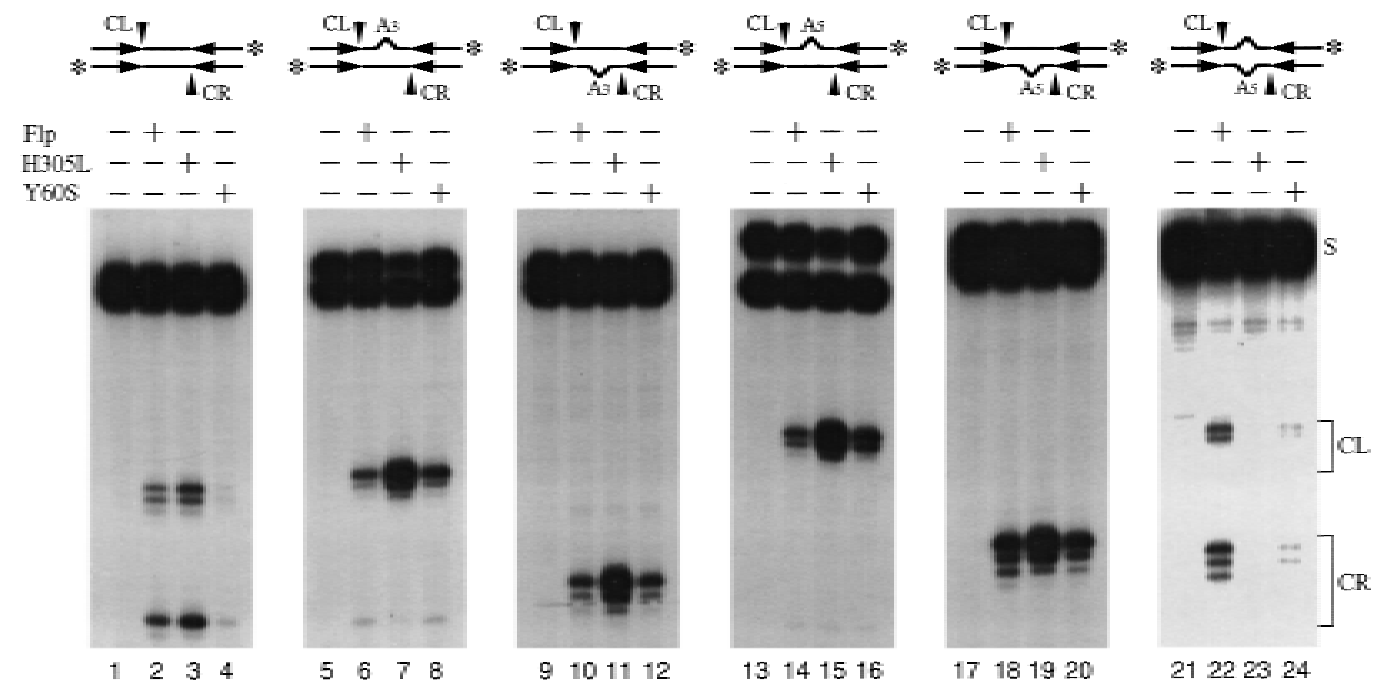

$91011+12$

13141516

$17 \quad 181920$

21222324

Figure 2. Flp-mediated strand cleavage in substrates containing $A_{3}$ - or $A_{5}$-spacer bulges. The two Flp-binding elements of the substrates (represented by the pair of parallel arrows in head-to-head orientation) have the same DN A sequence as those present in the $2 \mu$ plasmid, the native substrate for Flp. The bulges, when present within the spacer, are represented by the wedge shapes. The normal spacer has the sequence $5^{\prime}-$ TTTCTAGA- $^{\prime}$ ' and $3^{\prime}$-AAAGATCT $-5^{\prime}$ in the top and bottom strands, respectively. The bulges are placed between the fourth and fifth positions of the spacer, between $C$ and $T$ on the top strand and between $A$ and $G$ on the bottom strand. Therfore, the positioning of the top strand bulge with respect to the labile phosphate at the left is identical to the positioning of the bottom strand bulge with respect to the labile phosphate at the right. The short vertical arrows indicate the cleavage points on the top and bottom strands (at the left and right ends, respectively, of the spacer). The presence or absence of Flp or a Flp variant in a reaction is indicated by +or -above the corresponding lane. The asterisk $\left(^{*}\right)$ represents the $3^{\prime}$ end-label on the two strands. The substrate strands are denoted by $\mathrm{S}$. The cleavage products from the top and bottom strands are indicated by $C L$ and $C R$, respectively. The multiplicity in the cleavage bands (seen here and in subsequent figures) can be accounted for partly by some heterogeneity in the label ed substrate, and partly by the tendency of Flp to introduce occasional aberrant cleavages in linear substrates. 
the unbulged spacer, was restored with wild-type FIp (Fig. 2, lane 22). Flp(Y660S) was only weakly active on this substrate, as it was with the bulge-free substrate (Fig. 2, cf. lane 24 with lane 4). In contrast to its action on the bulged substrates, FIp(Y 60S) did not yi el d I arge cleavage disparity with the doubly bulged substrate. N o cleavage was detectable with $\mathrm{Flp}(\mathrm{H} 305 \mathrm{~L})$ on the substrate with the bal anced bulges (Fig. 2, Iane 23). This result was not unexpected, based on previous observations that $\mathrm{Flp}(\mathrm{H} 305 \mathrm{~L})$ was much less active relative to Flp on altered substrates such as half-sites, full-sites with mismatches adjacent to the cleavage positions, or full-sites containing mismatched insertions within the spacer (Serre et al. 1992; Whang et al. 1994; C. Harkey and M. Jayaram, unpubl.).

The strand cleavage data permit the following conclusions. By predisposing the FIp-binding arms of the DN A substrate to stack in a particular way, one active site for cleavage (e.g., the "top-strand cleavage pocket") can be assembled from the bound Flp dimer, whereas the second one (the "bottom-strand cleavage pocket") is effectively excluded (CL $\gg C R$; Fig. 2, lanes 6-8 and 14-16). On reversing the directionality of the stacking, the directionality of the strand cleavage bias can also be reversed ( $C R \gg C L$; Fig. 2, lanes 10-12 and 18-20). The inability of a bending-deficient Flp mutant to establish a catalytical ly productive dimer interface can be overcome by providing the mutant with substrates containing bulge-induced directed bends. It is important to note that the observed cleavage bias with the bulged substrates was the same for Flp as well as the joining-incompetent Flp(H305L). The failure to observe DN A cleavage at one of the scissile phosphates cannot therefore be attributable to a rapid reversal of the cl eavage reaction.

Strand cleavage within a bulged substrate by a catalytically complementing pair of Flp step-arrest mutants

The strong strand bias in cleavage observed with the bulged substrates is compatible with the shared architecture of the FIp active site (Fig. 3A,B; Chen et al . 1993; Pan et al. 1993). The top-strand bulges may promote a DNA configuration that permits functional alignment between the catalytic tyrosine (Tyr-343) of the right FIp monomer and the RHR cluster of the left FIp monomer, but not the other way round (Fig. 3A). Similarly, the bottom-strand bulges may foster a substrate configuration that only allows productive congregation of the triad cluster from the right monomer and Tyr-343 from the left monomer (Fig. 3B).

If the substrate structure is responsible for restricting FIp active site assembly, fruitful catal ytic complementati on between a triad mutant of FIp and FIp(Y 343F) will be possible if, and only if, each mutant occupies the correct binding arm on the bulged substrate. For the top-strand bulge, no cleavage is expected when the FIp(Y 343F) is stationed at the right, and the triad mutant is stationed at the left. Only the reverse placement of the two protein partners should yield strand cleavage. For the bottomstrand bulge, the expectation would be the opposite.

The protein replacement strategy described by Lee et al. (1994) was used to di rect catal ytically complementing mutant FIp partners to specific arms of the DNA substrates containing top- or bottom-strand bulges (Fig. 3C). The order of addition and the relative amounts of the mutant proteins [FIp(Y343F) first, followed by a relative excess of FIp(R191S, H305L)] would place the former on the normal Flp-binding arm and the latter on the weak Flp-binding arm (marked with an $X$ in Fig. 3) of a substrate. For each reaction set (Fig. 3, I-IV), the rightmost lane represents the targeted reaction and should be compared with the FIp reaction (second lane from the left) or the individual mutant reactions (third and fourth lanes from the left). The expected DNA associations for the mutant protein pair are diagrammed above each panel in Figure 3. The cleavage outcomes from these reactions matched the predictions of the "directional arm stacking" model. Note that only background levels of cleavage were seen when FIp(Y343F) occupied the right binding site of the substrate with top-strand bulge (Fig. 3, lane 5, panel I) or the left binding site of the substrate with bottom-strand bulge (Fig. 3, lane 20, panel IV). When the relative placement of this mutant was reversed on the two substrates, catalytic complementation was obtained, yielding strongly biased cleavage on the top or the bottom strand (Fig. 3, lane 10, panel II and Iane 15, panel III).

These results reaffirm the notion that half-of-the sites activity in wild-type FIp results directly from the positioning of the shared catal ytic resi dues, as determined by the spatial disposition of the Flp-bound DNA arms. In the mutant combination, with only one complement of the shared residues, assembly of the lone active site on a substrate with prepositioned DNA arms is specified by a unique FIp mutant-DNA association.

Active site exclusion: exclusion of the triad domain function or Tyr-343 function or both?

In principle, there are at least two obvious ways of effecting active site exclusion. One is by preventing the orientation of the scissile phosphate [by the triad domain of the FIp monomer situated adjacent to it; referred to as cis-activation by Lee and Jayaram (1993)] for nucleophilic attack by Tyr-343. The other is by misdirecting Tyr-343 of the Flp monomer bound across the spacer from its normal line of attack. A combination of both strategies is al so conceivable.

Once the phosphate at the recombination border has been oriented by a bound Flp monomer, it can be cleaved by an exogenous nucleophile, such as the peroxide anion derived from hydrogen peroxide or the phenolate moi ety derived from tyrosine mimics (Kimball et al. 1993; Lee and Jayaram 1993). The cleavage efficiency of a given nucleophile is limited by its nucleophilic strength, its accessibility to the reaction center, as well as its potential effects on protein structure. The reaction is absolutely dependent on phosphate activation as indi cated by 
C

A

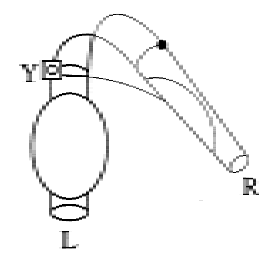

B

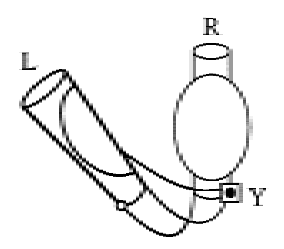

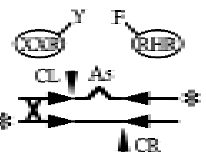

$\mathrm{Flp}-+-1 \mathrm{CR}$

r343F --+-1
S-H3065 --+1
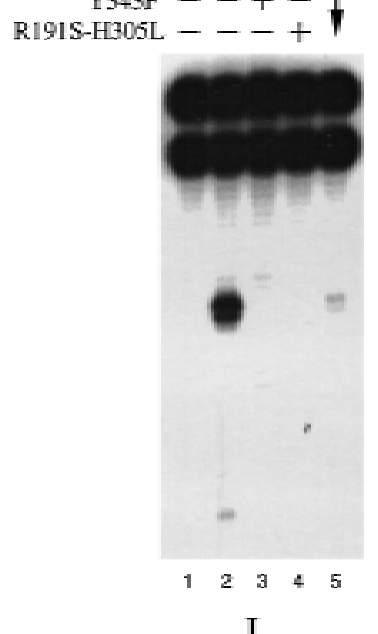

I

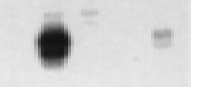

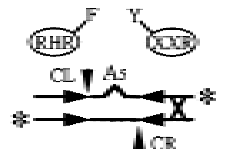

$-+---$

--+-+
---+1

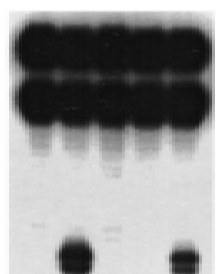

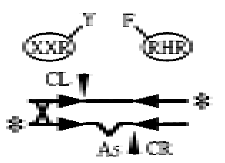
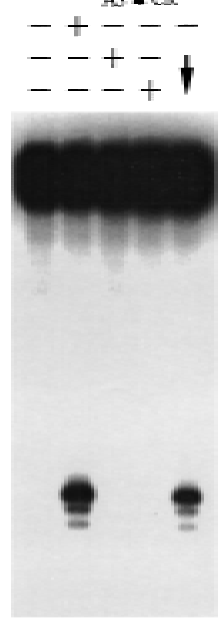

$\begin{array}{lllll}11 & 12 & 13 & 14 & 15\end{array}$

III

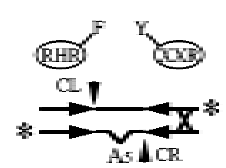

$$
-+--
$$$$
--+-1
$$$$
---+
$$

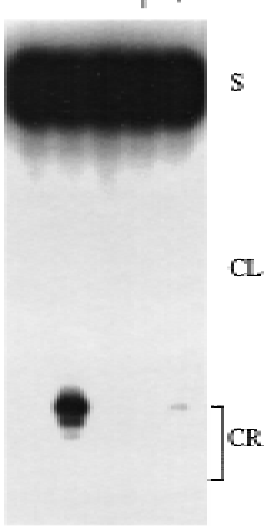

$1617 \quad 15 \quad 1920$

IV

Figure 3. The shared active site of Flp and active site exclusion: strand cleavage activity on targeted placement of Flp(Y343F) and Flp(R191S, H305L) on bulged substrates. (A,B) The constraints imposed by the DN A structure on the assembly of the shared active site of FIp are diagrammed schematically. The DN A arms ( $L$ for left; $R$ for right) are shown by a cylindrical representation, and the bound Flp monomers are indicated. When the DNA arms are tilted as shown in A, with the R arm extended below the plane of the paper, the active site is assembled at the phosphate on the left ( $O$ is al igned with the catalytic tyrosine, $Y$, donated by the right $F / p$ monomer). The labile phosphate on the right is not targeted for breakage in this configuration ( $(\mathbf{)})$. When the arms are tilted as shown in B, with the $L$ arm jutting above the plain of the paper, the shared active site can be assembled only at the labile phosphate on the right. The DN A arms are so positioned as to approximate the experimentally measured bend angle of $>140^{\circ}$ (Schwartz and Sadowski 1990; Chen et al. 1992a). However, the overall geometry of the bend depicted here is purely imagi nary. N ucl eotide bulges placed on one or the other strand at equival ent positions with respect to the scissile phosphodiester bonds can, in principle, force the FIp-occupied DN A arms to take up the configuration in A or that in B. The restricted arm dispositions would be consistent with the inference from N M R and FRET analyses (Rosen et al. 1992; Gholke et al. 1994) that the DNA arms flanking the bulge are tilted away from it. The active site configurations in A and B can account for the cleavage results obtained with substrates containing the top-and bottom-strand bulges, respectively (see Fig. 2). (C) The substrates containing spacer bulges in the top strand (I and II) or in the bottom strand (III and IV) were label ed at the $3^{\prime}$ ends (asterisks). An Flp-binding arm marked with an X carried a point mutation (from C-G to T-A at position 7) that causes relatively rapid recycling of a Flp monomer bound to it (Lee et al. 1994, 1996). Reactions (lanes 1,6,11,16) are controls to which neither Flp nor a Flp variant was added. The protein present in reactions (represented by lanes 2-4,7-9,12-14, and 17-19 is indicated by the + sign above them. Directed protein placement in the reactions (depicted in lanes $5,10,15,20$ ) was accomplished by the procedure described by Lee et al. (1994). First, both substrate arms were filled with Flp(Y 343F); the monomer bound to the X arm was then replaced by FIp(R191S, H305L). The resulting arrangements of the Flp mutant pair are indicated above panels I-IV. The cleavage products from the top and bottom strands are designated as CL and CR, respectively. (S) The label ed bands from the substrate.

the inability of Flp variants altered at Arg-191 or Arg-308 to assist peroxide cleavage (Lee and Jayaram 1993). We have taken advantage of the hydrogen peroxide reaction to ask whether, in a bulged substrate complexed with FIp, both phosphodiester bonds (on the top and the bottom strands) are susceptible to cleavage. The results are presented in Figure 4.

In the unbulged substrate (Fig. 4A, lanes 1-4), or in the one with balanced $A_{5}$ bulges (Fig. 4, lanes 5-8), $\mathrm{Flp}(\mathrm{Y} 343 \mathrm{~F}$ ) could not mediate cleavage on its own (Fig. 4, lanes 2,6$)$, but could so when provided with hydrogen peroxide (Fig. 4, Ianes 3,7). There was rough parity in the top- and bottom-strand cleavages $(\mathrm{CL}$ and $\mathrm{CR}$, respec- tively). Slight preferences for one cl eavage over the other, often depending on the sequence context of the spacer, have been observed for the normal FI p-mediated cl eavage reactions as well. Note that the hydrogen peroxide-induced cleavage was significantly less efficient relative to the FIp reaction (Fig. 4, cf. Ianes 4 and 8 with lanes 3 and 7 , respectively). Cleavage results from the substrate containing the $A_{5}$ bulge in the bottom strand are shown in Figure 4C (lanes 9-13). As expected from previous results (Fig. 2), nearly all of the cleavage yielded by Flp al one occurred on the bottom strand (CR; Fig. 4, Iane 12). In contrast, reaction with Flp(Y343F) plus hydrogen peroxide resul ted in top and bottom cleavages with strong bias 
Figure 4. Cleavage activity of an exogenous small nucleophile in normal and bulged substrates associated with FIp(Y343F) or Flp. The reactions were carried out with the substrates containing the normal spacer (A), balanced spacer bulges (B), an unbal anced spacer bulge in the bottom strand $(C)$, or an unbal anced spacer bulge in the top strand (D). Cleavage products were assayed using the radioactive label present at the $3^{\prime}$ ends (asterisks). The presence or absence of Flp or Flp(Y 343F) or of hydrogen peroxide in a reaction is indicated above the lane corresponding to it. The cleavage products from the top and bottom strands are marked $C L$ and $C R$, respectively. (S) The substrate strands.

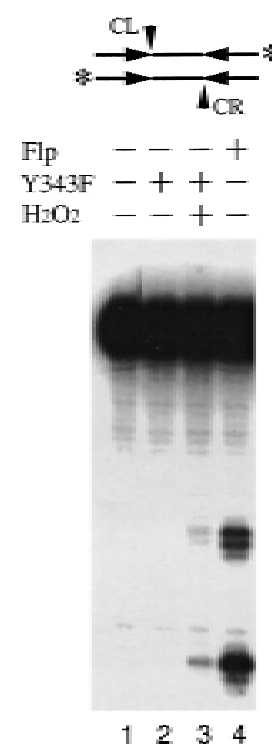

A
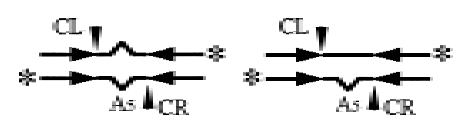

$--+$

$-++$

$-+-$

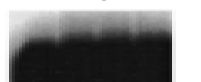

$--++$

$-++--$

$--++$

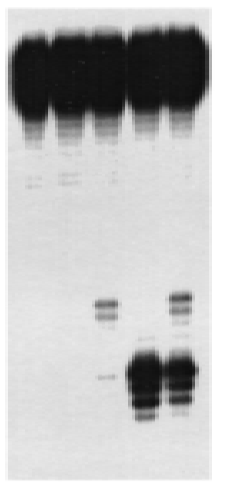

$910 \div 11213$

C

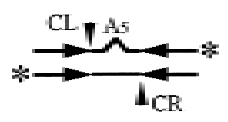

$---++$

$-++--$

$-+++$

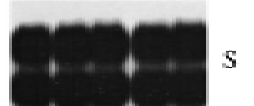

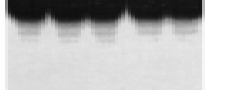

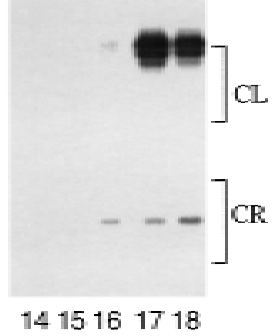

D toward top-strand cleavage ( $C L>C R$; Fig. $4 C$, lane 11 ). When hydrogen peroxide was included in the reaction with Flp, in addition to the expected bottom-strand cleavage, peroxide-mediated top-strand cleavage became apparent (Fig. 4, cf. lanes 13 and 12). When the experimental strategy underlying Figure $4 \mathrm{C}$ was applied to a substrate with an $A_{5}$ bulge on the top strand, the results were fully corroborative (Fig. 4D). In this case, the peroxide cleavage assisted by Flp(Y 343F) was biased toward the bottom strand (CR >CL; Fig. 4, lane 16). Cleavage by Flp al one occurred predominantly on the top strand (CL $\gg C R$; Fig. 4, Iane 17). In the Flp plus hydrogen peroxide reaction, the peroxide-mediated cleavage on the bottom strand was manifest over the background of Flpmediated cleavage (Fig. 4, Cf. CR in lanes 17 and 18). $\mathrm{N}$ ote that in Figure 4, CR in lane 18 increased over that in lane 17 despite a decrease in the extent of FIp cl eavage (cf. CL in lanes 17 and 18).

The ability of an exogenously supplied small nucleophile to mediate cleavage at the phosphodiester bonds in the bulged substrates that are refractory to cleavage by Tyr-343 shows that they are not excluded from their reactive orientation. Rather, it is the Tyr-343 of the Flp bound to one of the two substrate arms that is excluded from occupying its reactive position by the bulge. The weaker hydrogen peroxide reaction on the bottom strand and top strand, respectively, in the FIp(Y 343F) reactions in lanes 11 and 16 in Figure 4 (CR $<C L$ in Fig. 4C, lane 11; $C L<C R$ in Fig. 4D, lane 16), in the face of copious Tyr-343 reaction on these strands (CR $\gg C L$ in Fig. $4 C$, Iane 12; and CL $\gg C R$ in Fig. $4 D$, lane 17), is illuminating. We believe that, in the case of wild-type Flp, the special substrate configuration keeps the bottom-strand active site (in the case of the bottom bulge) or the topstrand active site (in the case of the top bulge) locked in its cleavage mode irreversibly, or nearly irreversibly. $\mathrm{Flp}(\mathrm{Y} 343 \mathrm{~F})$ would give rise to one of two frozen, but nonfunctional active sites on these substrates. Blockage of the RHR triad cleft by either Tyr-343 or Phe-343 may at least partially barricade the approach of hydrogen peroxide to the labile phosphates.

Effects of altering the RHR triad to Tyr-343 spacing on strand cleavage by Flp

The normal 8-bp spacing between the phosphodiester bonds that partake in cleavage/ exchange, as well as the 34-amino-acid spacing between Tyr-343 and Arg-308 of the RHR triad in Flp, is critical to the recombination reaction. When the phosphodiester spacing is increased or decreased by $2 \mathrm{bp}$ or more, there is essentially a complete loss of recombination activity (Broach et al. 1982; Senecoff and Cox 1986). Similarly, when short peptide insertions are placed between Arg-308 and Tyr-343, the resultant Flp variants fail to carry out strand cleavage (Evans et al. 1990). Both results can be accounted for by the misalignment between the RHR triad from one Flp monomer and Tyr-343 from its partner, as a result of perturbing either the enzyme structure or the substrate geometry. Is it possible then to compensate for a mislocated Tyr-343 and restore catalytic alignment by increasing the spacer flexibility of the substrate?

The ability of Flp variants harboring insertions of 3, 4, 10, and 15 amino acids between Arg-308 and Tyr-343 to mediate strand cleavage on substrates containing the normal spacer or "flexible" spacers is shown in Figure 5. Thethree-and four-resi due insertion variants of $\mathrm{Fl} p$ have been described previously (Evans et al. 1990; Chen et al. 1992b). The other two were constructed for this study and contained flexible peptide tethers that have been 


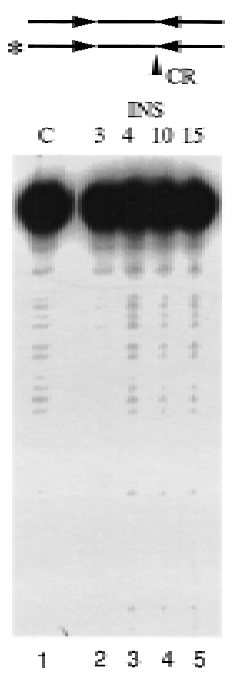

A
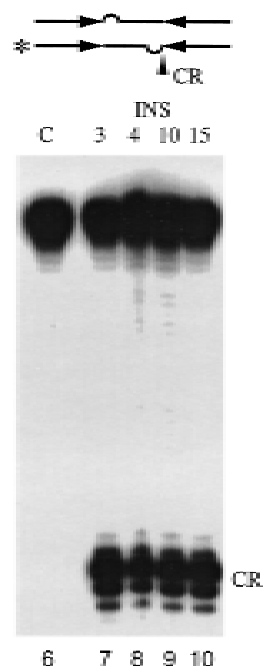

B

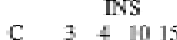

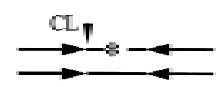

TWS

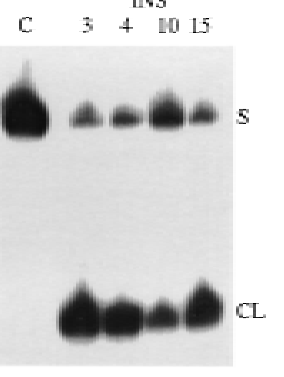

$\begin{array}{lllll}11 & 12 & 13 & 14 & 15\end{array}$

C
Figure 5. Effect of structural flexibility within the spacer on strand cleavage by Flp variants containing peptide insertions between Arg 308 and Tyr-343. The fully matched spacer (5'TTTCTAGA-3'/3'-AAAGATCT-5') within the normal substrate $(A)$ was deemed nonflexible. The spacer containing two mismatches at the ends $\left(5^{\prime}-\mathrm{TT}-3^{\prime} / 3^{\prime}-\mathrm{TT}-5^{\prime}\right.$ at the left and $5^{\prime}-$ CT $-3^{\prime} / 3^{\prime}-\mathrm{CT}-5^{\prime}$ at the right) (B) or that containing a nick on the top strand following $5^{\prime}-\mathrm{TTT}^{\prime} \mathrm{3}^{\prime}$ (C) were deemed flexible. The substrates in A and B were $3^{\prime}$ end-label ed on the bottom strands; those in C were $3^{\prime}$ end-label ed at the top strand nick within the spacer. The lanes marked $C$ refer to reaction mixtures containing the label ed substrate to which no Flp variant was added. The length of the peptide insertions in each of the FIp variants used in the cleavage assays is indicated above the appropriate lanes. The bands marked S, CR, and CL stand for the substrate, and the cleavage products from the bottom and top strands, respectively.

used previously to link two polypeptide domains (Huston et al. 1988). In the two types of flexible substrates used here, the native spacer length of 8 bp was maintained. Spacer flexibility was introduced either by placing two base mismatches at either end of the spacer (Fig. $5 \mathrm{~B}$, lanes $6-10$ ) or by placing a nick on the top strand three bases away from the labile phosphate (Fig. 5C, lanes 11-15). Although the normal substrate was not cleaved by the insertion variants (Fig. 5A, lanes 2-5), the flexiblesubstrates were all cleaved by these proteins (Fig. 5B, lanes 7-10; C, lanes 12-15). Furthermore, Tyr-343 was used as the nucleophile in these cleavages. N o cleavage was obtained when the insertion variants contained a phenylalanine substitution at position 343 (B.R. Evans, T. Tonozuka, and M. Jayaram, unpubl.). All three substrates were cleaved by wild-type Flp (data not shown).

The mi sal ignment and consequent inactivity of an improperly positioned protein nucleophile on a fully basepaired spacer, and the restoration of its alignment and activity on a nicked or a partially unpaired spacer, brings into focus the impact of DNA structure on molding the shared active site configuration of Flp.
Elimination of normal base pairing within the spacer DNA abolishes active site exclusion

Can we overcome the barrier to the si multaneous assembly of two active sites within a Flp dimer by completely removing the structural constraints caused by complementary base pairing of the spacer DNA? To test this idea, we reacted Flp with a substrate containing a fully unpaired spacer (an 8-nucl eoti de bubble) (Fig. 6A). Under these conditions, we not only observed the Flp cleavage product $\mathrm{CR}$, but also the hairpin product $\mathrm{H}$ resulting from the reductional recombination of a full-site (Fig. $6 \mathrm{~A}$, lane 4; see also B). Hairpin formation has not been observed with substrates containing the normal spacer (e.g., Fig. 6A, lane 2). This reaction requires a doublecleavage event within a single-substrate molecule-to generate the phosphotyrosine bond at one spacer end and to expose a 5'-hydroxyl group at the other spacer end (Fig. 6B). We do not know whether the two cleavage events are coincident or temporally separated. However, the formation of the hairpin demonstrates that rel ease of Tyr-343 from covalent bondage to a cleaved DN A strand (effectively the disassembly of one active site) is not a prerequisite for cleavage of the other DNA strand (the assembly of the second active site).

Although the data shown in Figure 6A is from a denaturing gel, the authenticity of the hairpin was established by electrophoresis in native gel as well (data not shown). The native gel analysis excluded the possibility that the product $\mathrm{H}$ could have arisen by an intermolecular event, by the cleaved strand from one DN A molecule attacking the phosphotyrosine bond formed in a second molecule. Furthermore, a reaction containing mixture of $\mathrm{Flp}(\mathrm{Y} 343 \mathrm{~F})$ and $\mathrm{FIp}(\mathrm{R} 191 \mathrm{~S}, \mathrm{H} 305 \mathrm{~L})$ yielded the cleavage product CR, but produced no hairpin (data not shown). Because the mutant Flp pair has the catalytic complements to assemble only one active site from a di mer (one good RHR triad domain and one Tyr-343), double cleavages within a single molecule of the bubbled substrate would have been impossible.

The reductional recombinase activity of Flp, manifested only on the fully unpaired spacer, implies that there is little or no contribution by the DN A-bound FIp dimer, per se, to active site exclusion. This phenomenon is mediated entirely by the structural geometry and base complementarity of the spacer DNA.

\section{Discussion}

The double-hel ical configuration of DNA, together with the topography of the reaction complex containing two substrate and four recombinase molecules, poses a serious chal lenge to the precision and coordination of strand breakage and joining during conservative site-specific recombination. The experiments reported here reveal how DNA-induced relative positioning of the protein monomers can be used to achieve spatial selectivity and temporal separation of the relevant phosphoryl transfer steps. We discuss below features of the specific solution devised by Flp and expl ore its general implications in the Int family recombination mechanism. 
Figure 6. Reductional recombination mediated by Flp within a DNA substrate containing a completely unpaired spacer. (A) The reaction contained either the normal substrate with the fully complementary 8-bp spacer (lanes 1,2) or the abnormal substrate with a fully mismatched spacer (8-nucleotide bubble; lanes 3,4). The nucleotide sequence of the spacer bubble was 5'-TTTTAAAA-3'/3'-TTTTAAAA $-5^{\prime}$. The substrates were label ed on the bottom strand at the $3^{\prime}$ end (asterisks). The substrate band is indicated by $\mathrm{S}$ and the cleavage product by $\mathrm{CR}$. $\mathrm{H}$ is the hairpin product resulting from reductional recombination. (B) The reaction pathway that leads to the formation of $\mathrm{H}$ is diagrammed. An essential feature of the reductional recombination is a double-stranded cleavage event within a substrate.

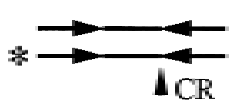

$$
\text { Flp }
$$
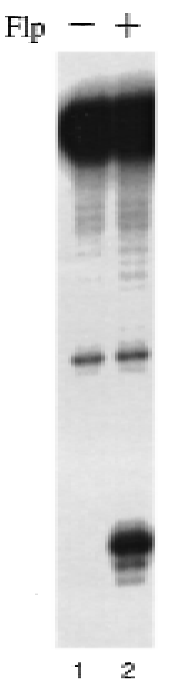

The role of DNA in assembling the shared active site of Flp

The Flp solution has two parts to it. First, the functional components of one Flp active site are situated in two separate Flp monomers (Chen et al. 1992b). Within an oligomer of Flp, then, spatial selection of active sites becomes possible by specifying the monomer pairs that establish a productive dimer interface (Lee et al. 1996). Temporal separation of active sites becomes possible if the disassembly of one dimer interface can be associated with the assembly of an equivalent, second functional interface.

In accommodating the catalytic contributions by two Flp monomers bound on either side of the spacer (in opposite orientation and on approximately opposite faces of the helix) towards active site assembly, some structural contortion of the DN A substrate within the spacer can be expected. The anomolously slow electrophoretic mobility of Flp-DN A complexes in polyacrylamide suggested that a FIp dimer introduces a strong bend of $>140^{\circ}$ within the spacer (Schwartz and Sadowski 1990; Chen et al. 1992a). We do not know whether this inferred shortening of the end-to-end distance in DNA is attributable to a unique bend or a composite of more than one bend, or whether the bent DN A axis is planar or writhed. N evertheless, the Flp-induced DN A bend is directly relevant to catalysis, as point mutants that fail to induce this bend are inactive in recombination (Schwartz and Sadowski 1989; Chen et al. 1991; Kulpa et al. 1993). In a simple scenario of the natural situation, one may imagine that a Flp dimer can yiel d only one of two active sites at a time because it can establish only one of two equivalent asymmetric bends at a time. Our results suggest strongly that predisposition of the DNA arms in a bulged
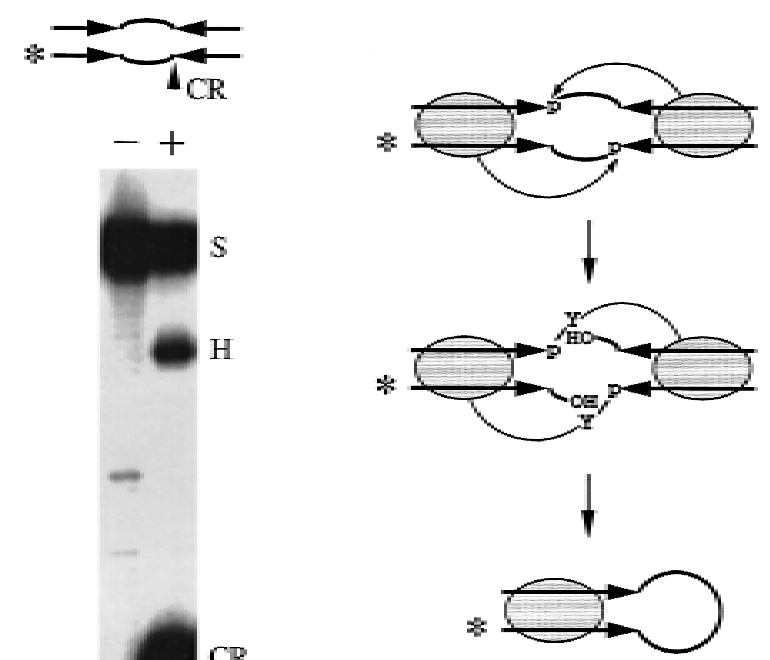

$\mathrm{H}$

B

substrate imposes active site selectivity by trapping one configuration of the bent FIp-DN A complex, and excluding the other. Whereas the bulge-induced DNA bend may not be identical to that induced by FIp during normal recombination, the bulge functions as a "geometric filter" that imposes the bend choice on FIp. Luetke and Sadowski (1995) noticed that, in cl eaved FIp-DN A complexes, the center and direction of DNA bends were distinct for top- and bottom-strand cleavages. Although their DNA substrates and ours cannot be compared directly (because of the difference in the number of Flpbinding elements harbored by them), the experimental outcomes in both cases fit into a common interpretation-the assembly or nonassembly of a FIp active site is determined by the relative disposition of two Flp-bound DNA arms. This interpretation also agrees with the observation that a three-armed DNA junction (a $Y$ structure) or a Holliday junction with only three good Flpbinding arms can be resolved by Flp into a normal recombinant plus a hairpin molecule or into two normal recombinants, respectively (Qian and Cox 1995; Lee et al. 1996). The resolution of a three- or four-armed junction requires the cooperative action of two active sites (for breakage and exchange at two labile phosphates). Therefore, the conformational freedom of the DN A arms can give rise to FIp-FIp interactions that yield two active sites from either three or four FIp monomers.

Switching of the recombination complex from the Holliday-forming mode to the Holliday-resolving mode

Our results demonstrate that al ternative alignments of the DNA arms, as diagrammed in Figure 7A-D, can ac- 
A

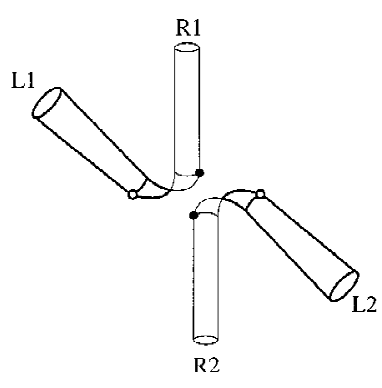

C

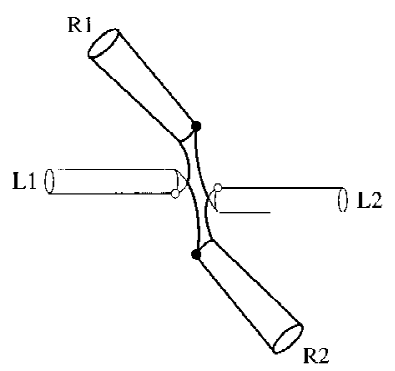

B

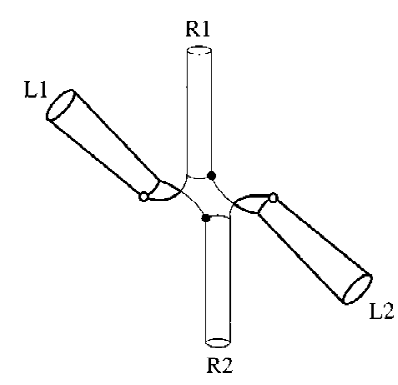

$\mathbf{D}$

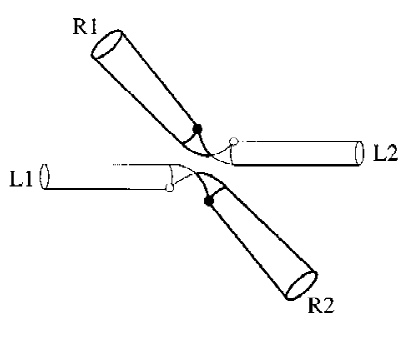

Figure 7. Selective active site exclusion at either spacer end within the recombination complex accounts for the two-step strand-exchange mechanism by Flp. The model outlines the contribution of active site exclusion to the mechanism of FIp recombination. The cylindrical representation of the DNA arms of the Holliday junctions is analogous to that used by Pohler et al. (1996). (A) The stacking of the substrate arms as shown here permits active site assembly at the scissile phosphates indicated by the filled circles (adjacent to arms R1 and R2, the right arms). In this arrangement, the FIp monomers bound to R1 and R2 are the donors of the RHR triad, and those bound to L1 and L2 (the left arms) are the donors of Tyr-343. Note that the relative placement of the DN A arms of the two substrates roughly mimics an antiparallel configuration ( $L 1$ is disposed away from L2 and R1 is positioned away from R2). No active sites can be assembled at the phosphates shown by the open circles (adjacent to $L 1$ and $L 2)$. (B,C) The first pair of strand exchanges $(A \rightarrow B)$ results in the Holliday junction shown in B. Flipping of the DNA arms coupled with strand swapping within the spacer isomerizes the junction $(B \rightarrow C)$. The two functional active sites engage the phosphates adjacent to $L 1$ and $L 2$. The first pair of active sites (responsible for the formation of the Holliday intermediate) have been dismantled. In this configuration, the FIp monomers bound to $L 1$ and $L 2$ are the triad donors; those bound to R1 and R2 are the Tyr-343 donors. (D) The second pair of strand exchanges $(C \rightarrow D)$ resolves the Holliday intermediate into the reciprocal recombinants L1-R2 and L2-R1.

count for the Holliday-forming and Holliday-resolving steps of FIp recombination. The chemical steps $A$ and $C$ and the isomerization step $B \leftrightarrow C$ are likely coupled to the exchange of the spacer DNA between the recombination partners (see Fig. 7). Whereas all four scissile phosphates are oriented by the bound Flp monomers in the recombination complex, only two undergo breakage at one time, because only two tyrosine nucleophiles are positioned in their correct line of attack. This impediment posed by the normal DNA structure dictates that misalignment of the initially reactive tyrosines must precede the al ignment of the other two in their reactive orientation. The functional correspondence between Tyr-343 of a Flp monomer and its target phosphate (or the RHR triad of its partner monomer), which is a critical feature of the shared active site, can be destroyed by perturbations in the DNA or in the protein structure. However, provided the DNA is sufficiently flexible, altered protein structures can be tolerated. And, in the extreme case, when the DN A resistance is nearly absent, double-stranded DNA cleavage within a substrate be comes possible, as dismantling of the first active site is no longer a prerequisite for the assembly of the second one (absence of active site exclusion).

It must be noted that our experiments only address the chemical competence and orientation of a FIp di mer on a single DNA molecule and not of a Flp tetramer on two paired-DNA molecules. Do the DNA-protein interactions within the synaptic structure constrain cleavages to be paired at one or the other end of the spacer? All such pai red cl eavages can, in principle, be channel ed into the recombination pathway. It is conceivable that protein-protein interactions between Flp monomers bound on substrate partners can mediate end-specificity, as well as synchrony, of assembly and disassembly of active site pairs. Alternatively, is strand cleavage on one DNA molecule independent of strand cleavage on the synapsed partner? In this case, only a fraction of the cleavages will be recombination-proficient. Provided the breakage-joining reaction is in rapid dynamic equilibrium, appreciable rates of recombination can be achieved even in the midst of a significant proportion of futile events. Because we are largely unaware of the DNAprotein configuration of the synaptic structure, we cannot presently tackle these finer aspects of the recombination mechanism.

\section{Active site exclusion within the Int family}

Because all of the well characterized Int family recombinases ( $\lambda$ Int, Flp, Cre, and XerC / XerD) mediate recombination via a Holliday-junction intermediate, it is reasonable to surmise that active site exclusion must be a general phenomenon within this family. The shared active site and the trans mode of DN A cleavage are central elements in the mechanism of active site exclusion by Flp. In other members of this family, however, except for Flp-like yeast recombinases, the Cre protein and the $\lambda$ Int protein in one particular instance (Han et al. 1993; Yang and Jayaram 1994; Shaikh and Sadowski 1997), there is no direct evidence for a shared active site. In fact, evidence strongly favors cis DNA cleavage by XerC/ XerD and by Int under most experimental situations (Nunes-Duby et al. 1994; Arciszweska and Sherratt 1995). The recently solved crystal structure of Cre complexed with DNA has posed a paradox by revealing cis cleavage (Guo et al. 1997), therefore directly contradicting the results from solution assays (Shaikh and Sad- 
owski 1997). cis-cleavage, per se, does not rule out a shared active site; it only means that the catalytic tyrosine is not among the shared residues. Even if an active site were contained entirely within one recombinase monomer, the functionality of this active site could be dependent on allosteric interactions between monomers bound across the strand-exchange region. Experiments with the Int system strongly imply that such "crosscore" interactions are critical to the efficiency of resolution of Holliday junctions by Int (Kho and Landy 1994). Therefore, the operation of geometric filters, constituted by recombinase-DNA and recombinase-recombinase interactions, during active site assembly is likely to be a common feature within the Int family.

\section{Materials and methods}

\section{Flp and Flp variants}

The step-arrest mutants of Flp, Flp(Y343F), and FIp(R191S, $\mathrm{H} 305 \mathrm{~L}$ ) have been described previously (Chen et al. 1992b; Lee et al. 1994). Construction of Flp variants containing 3- or 4amino-acid insertions between the RHR triad and Tyr-343 has been detailed by Evans et al. (1990). The 10- and 15-amino-acid insertions were obtained by placing synthetic oligonucleotides into the Ball site that straddl es the codons for Val-338, Ala-339, and Arg-340 within the FLP gene, thereby altering the spacing between Val-338 and Ala-339. The sequences of the peptide inserts were Gly $_{4}-$ Ser-Gly ${ }_{4}$ Ser and Gly $_{4}-$ Ser-Gly ${ }_{4}-$ Ser-Gly ${ }_{4}-$ Ser. Wild-type Flp and Flp variants used in the assays described here were purified to $90 \%-95 \%$ homogeneity by an affinitypurification protocol (Parsons et al. 1988).

\section{Synthetic recombination sites}

Oligodeoxynucleotides for construction of full-site substrates were synthesized in an Applied Biosystems DNA synthesizer (model 380A) using phosphoramidite chemistry (Beaucage and Caruthers 1981). Hybridization of appropriate oligonucleotides was carried out as described previously (Lee et al. 1996). The rel evant features of the various substrates used are described in the Results section, displayed in the figures, and explained in their legends. Nonspecific nucleotide segments of specific length that have no effect on Flp-mediated reactions were present on either side of the Flp recombination target site. The lengths of the strands were so chosen as to distinguish the products resulting from alternative strand-cleavage events. In substrates, where directed protein replacement was required, the appropriate Flp-binding elements were weakened by altering a critical Flp contact position within each of them from a C-G to an T-A pair (Lee et al. 1994). The complete sequences of the substrates are available on request.

The $3^{\prime}$ end of an oligodeoxynucleotide was labeled with $3^{\prime}$ $\left[\alpha-{ }^{32} \mathrm{P}\right]$ cordycepin $5^{\prime}$-triphosphate using the terminal transferase reaction. The unreacted cordycepin phosphate was removed by spin dialysis on a G-25 column. Hybridization to the partner oligodeoxynucleotide was done in TE buffer.

\section{Strand-cleavage/strand-transfer assays}

The strand-cleavage reactions were done under standard recombination conditions (Chen et al. 1992b). N ormally, 0.05 pmole of the $3^{\prime}$ end-labeled substrate was reacted with $\sim 0.5$ pmole Flp or Flp variant ( $\sim 5$ pmoles of Flp per pmole-binding element) in $30 \mu \mathrm{l}$ of the reaction mixture. Incubations were done at $30^{\circ} \mathrm{C}$ for
$10 \mathrm{~min}$. Reactions were stopped by addition of SDS $(0.1 \%$ final concentration) and treated with proteinase $\mathrm{K}$ ( $100 \mu \mathrm{g}$ per sample for $1 \mathrm{hr}$ at $37^{\circ} \mathrm{C}$ ). Samples were processed further and analyzed by el ectrophoresis in denaturing polyacrylamide gels (Lee et al. 1996).

Strand cleavage by hydrogen peroxi de was done as outlined by Lee and Jayaram (1993).

\section{Directed protein replacement on synthetic Flp substrates}

The protocol for targeting a Flp variant to a given Flp-binding arm of the substrate has been described by Lee et al. (1994). First, all binding arms were saturated with FIp(Y343F) at a molar ratio of the protein to binding element of approximately 8:1. This incubation was done at $30^{\circ} \mathrm{C}$ for $10 \mathrm{~min}$ in recombination medium (Chen et al. 1992b) containing $130 \mathrm{~mm} \mathrm{~N} \mathrm{aCl}$. An eightfold excess of the triad variant Flp(R191S, H305L) was added to the assay mixture at time zero. The higher relative concentration of protein would result in the sel ective transplacement of Flp(Y 343F) by Flp(R191S, H305L) on the weak binding arms. Strand cleavage was assayed at $10 \mathrm{~min}$ from the addition of the second Flp variant. The final $\mathrm{N} \mathrm{aCl}$ concentration in the reaction was $150 \mathrm{~mm}$.

\section{General methods}

Restriction enzyme digestions, isolation of plasmid DNA, and other miscellaneous procedures were done as described by Sambrook et al. (1989).

\section{Acknowledgments}

This work was supported principally by a grant from the $\mathrm{Na}$ tional Institutes of Health. Partial support was received from the Robert F. Welch Foundation. Cecil Harkey provided excellent technical assistance. We acknowledge helpful comments from David Sherratt and others on an earlier version of this manuscript.

The publication costs of this article were defrayed in part by payment of page charges. This article must therefore be hereby marked "advertisement" in accordance with 18 USC section 1734 solely to indicate this fact.

\section{References}

Abremski, K. and R.H. Hoess. 1992. Evidence for a second conserved arginine in the integrase family of recombination proteins. Protein Eng. 5: 87-91.

Arciszewska, L.K. and D.J. Sherratt. 1995. Xer site-specific recombination in vitro. EMBO J. 14: 2112-2120.

Argos, P., A. Landy, K. Abremski, J.B. Egan, E. HaggardLjungquist, R.H. Hoess, M.L. Kahn, B. Kalionis, S.V.L. Narayana, L.S. Pierson III, N. Sternberg, and J.M. Leong. 1986. The Integrase family of site-specific recombinases: Regional similarity and global diversity. EMBO J. 5: 433-440.

Beaucage, S.L. and M.H. Caruthers. 1981. Deoxynucleoside phosphoramidites: A new class of key intermediates for deoxypolynucleotide synthesis. Tetrahedron Lett. 22:18591862.

Broach, J.R., V.R. Guarascio, and M. Jayaram. 1982. Recombination within the yeast plasmid $2 \mu \mathrm{m}$ circle is site-specific. Cell 29: 227-234.

Chen, J.W., B.R. Evans, L. Zheng, and M. Jayaram. 1991. Tyr60 variants of Flp recombinase generate conformationally altered protein-DNA complexes: Differential activity in full- 
site and half-site recombinations. J. Mol. Biol. 218: 107-118.

Chen, J.W., B.R. Evans, H. Rosenfeldt, and M. Jayaram. 1992a. Bending-incompetent variants of Flp recombinase mediate strand transfer in half-site recombinations: Role of DNA bending in recombination. Gene 119: 37-48.

Chen, J.W., J. Lee, and M. Jayaram. 1992b. DNA cleavage in trans by the active site tyrosine during Flp recombination: Switching protein partners before exchanging strands. Cell 69: 647-658.

Chen, J.W., S.H. Yang, and M. Jayaram. 1993. Tests for the fractional active-site model in Flp site-specific recombination: Assembly of a functional recombination complex in half-site and full-site strand transfer. J. Biol. Chem. 268: 14417-14425.

Evans, B.R., J.W. Chen, R.L. Parsons, T.K. Bauer, D.B. Teplow, and M. Jayaram. 1990. Identification of the active site tyrosine of FIp recombinase: Possible relevance of its location to the mechanism of recombination. J. Biol. Chem. 265: 1850418510.

Gohlke, C., A.I. Murchie, D.M. Lilley, and R.M. Clegg. 1994. Kinking of DNA and RNA helices by bulged nucleotides observed by fluorescence resonance energy transfer. Proc. Natl. Acad. Sci. 91: 11660-11664.

Guo, F., D.N. Gopal, and G.D. Van Duyne. 1997. Structure of Cre recombinase complexed with DNA in a site-specific recombination synapse. Nature 389: 40-46.

Han, Y.W., R.I. Gumport, and J.F. Gardner. 1993. Complementation of bacteriophage lambda integrase mutants: Evidence for an intersubunit active site. EMBO J. 12: 4577-4584.

Huston, J.S., D. Levinson, M. M udgett-Hunter, M.S. Tai, J. N ovotny, M.N. M argolies, R.J. Ridge, R.E. Bruccoleri, E. Haber, R. Crea, and H. Oppermann. 1988. Protein engineering of antibody binding sites: Recovery of activity in an anti-digoxin single-chain Fv anal ogue produced in Escherichia coli. Proc. Natl. Acad. Sci. 85: 5879-5883.

Jayaram, M. 1994. Mechanism of site-specific recombination: The Flp paradigm. In Nucleic acids molecular biology (ed. F. Eckstein and D.M.J. Lilley), pp. 268-286. Springer-Verlag, Berlin, Germany.

Kho, S.H. and A. Landy. 1994. Dissecting the resolution reaction of lambda integrase using suicide Holliday junction substrates. EMBO J. 13: 2714-2724.

Kimball, A.S., J. Lee, M. Jayaram, and T.D. Tullius. 1993. Sequence-specific cleavage of DNA via nucloephilic attack of hydrogen peroxide, assisted by FIp recombinase. Biochemistry 32: 4698-4701.

Kimball, A.S., M.L. Kimball, M. Jayaram, and T.D. Tullius. 1995. Chemical probe and missing nucleoside analysis of FIp recombinase bound to the recombination target sequence. Nucleic Acids Res. 123: 3009-3017.

Kulpa, J., J.E. Dixon, G. Pan, and P.D. Sadowski. 1993. Mutations of the FLP recombinase gene that cause a deficiency in DNA bending and strand cleavage. J. Biol. Chem. 268: 11011108.

Lee, J. and M . Jayaram. 1993. M echanism of site-specific recombination: Logic of assembling recombinase catalytic site from fractional active sites. J. Biol. Chem. 268: 17564-17579.

Lee, J., I. Whang, and M. Jayaram. 1996. Assembly and orientation of Flp recombinase active sites on two-, three- and fourarmed DNA substrates: Implications for a recombination mechanism. J. Mol. Biol. 257: 532-549.

Lee, J., I. Whang, J. Lee, and M. Jayaram. 1994. Directed protein replacement in recombination full sites reveals trans-horizontal DNA cleavage by $\mathrm{Flp}$ recombinase. EMBO J. 13: 5346-5354.

Luetke, K.H. and P.D. Sadowski. 1995. The role of DN A bending in Flp-mediated site-specific recombination. J. Mol. Biol.
251: 493-506.

N unes-Duby, S.E., R.S. Tirumalai, L. Dorgai, E. Yagil, R.A. Weisberg, and A. Landy. 1994. Lambda integrase cleaves DNA in cis. EMBO J. 13: 4421-4430.

Pan, G., K. Luetke, and P.D. Sadowski. 1993. Mechanism of cleavage and ligation by the Flp recombinase: Classification of mutations in the Flp protein using in vitro complementation analysis. Mol. Cell. Biol. 13: 3167-3175.

Panigrahi, G.B. and P.D. Sadowski. 1994. Interaction of the $\mathrm{NH}$ 2- and $\mathrm{COOH}$-terminal domains of the FLP recombinase with the FLP recognition target sequence. J. Biol. Chem. 269: 10940-10945.

Parsons, R.L., P.V. Prasad, R.M. Harshey, and M. Jayaram. 1988. Step-arrest mutants of FIp recombinase: Implications for the catalytic mechanism of DNA recombination. Mol. Cell. Biol. 8: 3303-3310.

Pohler, J.R.G., M.-J.E. Giraud-Panis, and D.M.J. Lilley. 1996. T4 endonucl ease VII sel ects and al ters the structure of the fourway DNA junction: Binding of a resolution-defective mutant enzyme. J. Mol. Biol. 260: 678-696.

Qian, X.H. and M.M. Cox. 1995. Asymmetry in active complexes of FLP recombinase. Genes \& Dev. 9: 2053-2064.

Qian, X.H., R.B. Inman, and M.M. Cox. 1990. Protein-based asymmetry and protein-protein interactions in FLP recombinase-mediated site-specific recombination. J. Biol. Chem. 265: 21779-21788.

Rosen, M.A., L. Shapiro, and D.J. Patel. 1992. Solution structure of a trinucleotide A-T-A bulge loop within a DNA duplex. Biochemistry 31: 4015-4026.

Sadowski, P.D. 1995. The Flp recombinase of the 2-micron plasmid of Saccharomyces cerevisiae. Prog. Nucleic Acid Res. Mol. Biol. 51: 53-91.

Sambrook, J., E.F. Fritsch, and T. Maniatis. 1989. Molecular cloning: A laboratory manual. Cold Spring Harbor Laboratory Press, Cold Spring Harbor, NY.

Schwartz, C.J. and P.D. Sadowski. 1989. Flp recombinase of the $2 \mu \mathrm{m}$ plasmid of Saccharomyces cerevisiae bends its DNA target: Isolation of FIp mutants defective in DN A bending. J. Mol. Biol. 205: 647-658.

Schwartz, C.J. and P.D. Sadowski. 1990. FLP protein of $2 \mu \mathrm{m}$ circle plasmid of yeast induces multiple bends in the FLP recognition target site. J. Mol. Biol. 216: 289-298.

Senecoff, J.F. and M.M. Cox. 1986. Directionality in FLP protein-promoted site-specific recombination is mediated by DN A-DN A pairing. J. Biol. Chem. 261: 7380-7386.

Serre, M.C., B.R. Evans, H. Araki, Y. Oshima, and M. Jayaram. 1992. Half-site recombinations mediated by yeast-site specific recombinases FIp and R. J. Mol. Biol. 225: 621-642.

Shaikh, A.C. and P.D. Sadowski. 1997. The Cre recombinase cleaves the lox site in trans. J. Biol. Chem. 272: 5695-5702.

Stark, W.M., M.R. Boocock, and D.J. Sherratt. 1992. Catalysis by site-specific recombinases. Trends Genet. 8: 432-439.

Whang, I., J. Lee, and M. Jayaram. 1994. Active-site assembly and mode of DNA cleavage by Flp recombinase during fullsite recombination. Mol. Cell. Biol. 14: 7492-7498.

Yang, S.H. and M. Jayaram. 1994. Generality of the shared active site among yeast family site-specific recombinases: The R site-specific recombinase foll ows the Flp paradigm. J. Biol. Chem. 269: 12789-12796. 


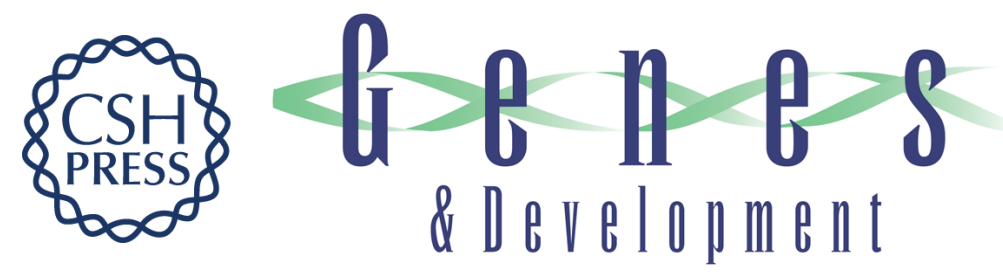

\section{Mechanism of active site exclusion in a site-specific recombinase: role of the DNA substrate in conferring half-of-the-sites activity}

Jehee Lee, Takashi Tonozuka and Makkuni Jayaram

Genes Dev. 1997, 11:

Access the most recent version at doi:10.1101/gad.11.22.3061

References

This article cites 39 articles, 15 of which can be accessed free at:

http://genesdev.cshlp.org/content/11/22/3061.full.html\#ref-list-1

License

Email Alerting

Receive free email alerts when new articles cite this article - sign up in the box at the top

Service right corner of the article or click here.

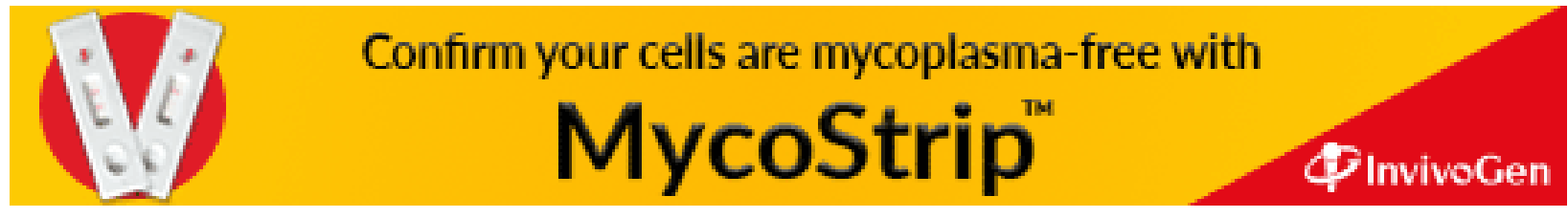

